\title{
SURGICAL CORRECTION OF AORTIC ARCH HYPOPLASIA ASSOCIATED WITH CRESTLIKE PROTRUSION OF THE SUPERIOR ARCH WALL AND ISTHMIC COARCTATION
}

\author{
Afksendiyos Kalangos, MD, PhD, ${ }^{\mathrm{b}}$ Jean Rubay, MD, PhD, ${ }^{\mathrm{a}}$ Ruth Ouaknine, MD, ${ }^{\mathrm{b}}$ Nicolas Murith, MD, ${ }^{\mathrm{b}}$ Laurence Cohen, MD, \\ and Yves Lecompte, MD, ${ }^{\mathrm{b}}$ Brussels, Belgium, and Massy, France
}

Aortic arch hypoplasia with varying degrees of severity is frequently seen in infants who have isthmic coarctation. In some cases of severe aortic arch hypoplasia, the superior aortic wall between the origins of the arch vessels becomes invaginated into the lumen like a crest. Extended end-to-end anastomosis can produce only limited relief of the arch hypoplasia. Although optimal surgical management of infants with aortic coarctation and concomitant hypoplastic arch remains controversial, we recognize, on the basis of our experience, that any persistent aortic arch stenosis after surgery can increase the prevalence of chronic systemic arterial hypertension in these patients. For this reason, we strongly believe that aggressive surgical enlargement of the hypoplastic arch segment at the time of infant coarctation repair to restore a homogeneous aortic arch like a "romanesque arch" is mandatory. We describe herein a modification of the technique previously reported by Amato, Rheinlander, and Cleveland, ${ }^{1}$ which provides an excellent option to the surgeon for the management of arch hypoplasia associated with crestlike protrusion of superior aortic arch wall.

Patients. From January 1997 to December 1999, 80 patients underwent surgical repair of aortic coarctation and associated aortic arch hypoplasia. In 36 of these patients, the diameter of the distal aortic arch was less than $30 \%$ of that of the descending aorta, and preoperative echocardiographic

From the University Hospital of Saint-Luc, Catholic University of Louvain, ${ }^{\mathrm{a}}$ Brussels, Belgium, and the Unit of Pediatric and Prenatal Cardiology, Institut Cardiovasculaire Paris Sud, ${ }^{\mathrm{b}}$ Massy, France.

J Thorac Cardiovasc Surg 2001;121:996-8

Copyright () 2001 by The American Association for Thoracic Surgery

$0022-5223 / 2001 \$ 35.00+0 \quad \mathbf{1 2 / 5 4 / 1 1 2 8 1 9}$

doi:10.1067/mtc.2001.112819
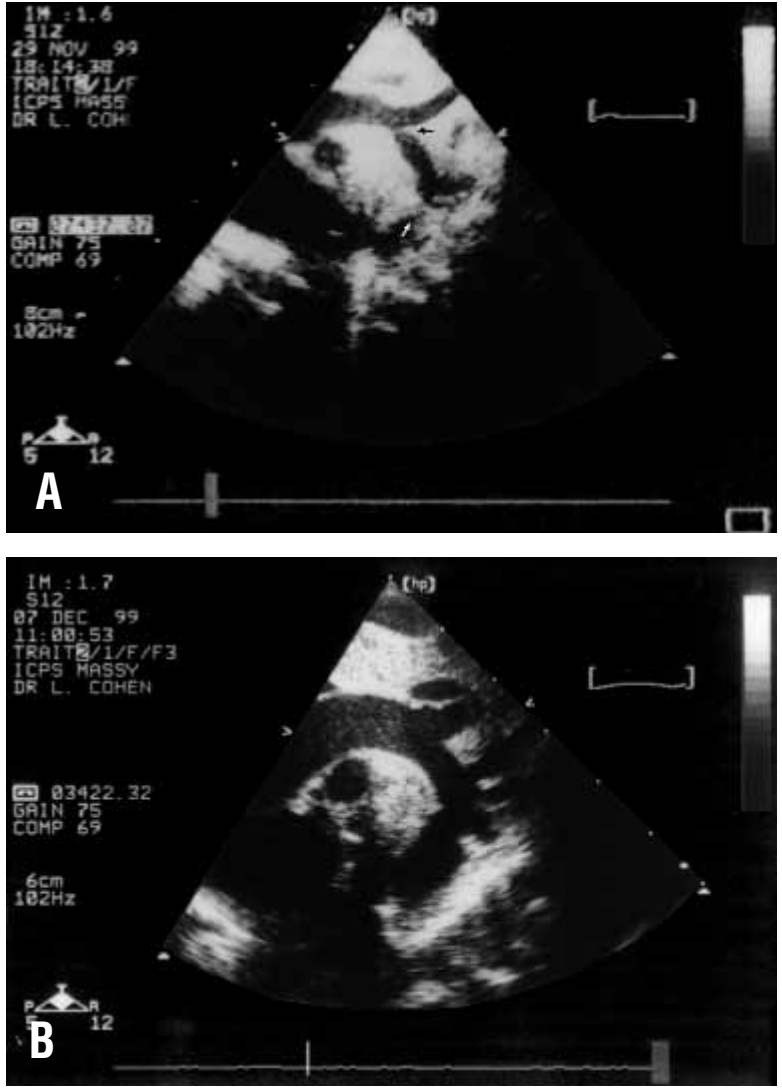

Fig 1. Echocardiographic image of crestlike protrusion of the superior aortic wall between the origin of the left common carotid and left subclavian arteries in a hypoplastic distal aortic arch (black arrow) associated with isthmic coarctation (white arrow) before surgery (A) and the reconstructed aortic arch after surgery $\mathbf{( B )}$. 


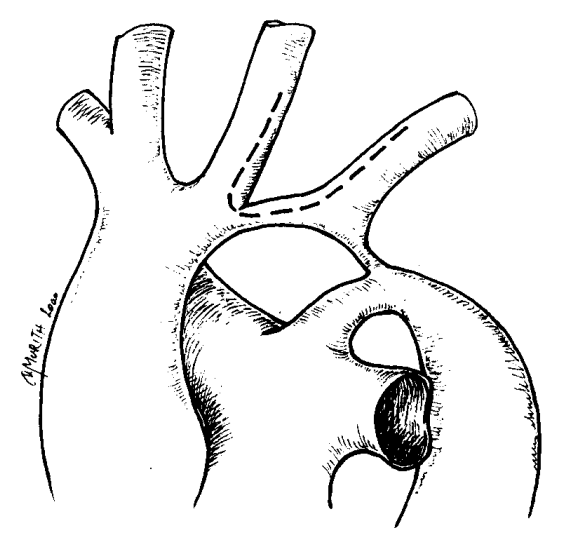

A

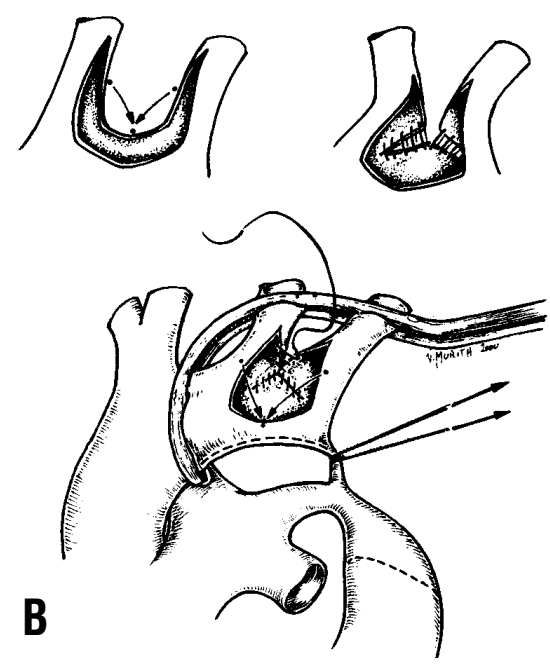

C

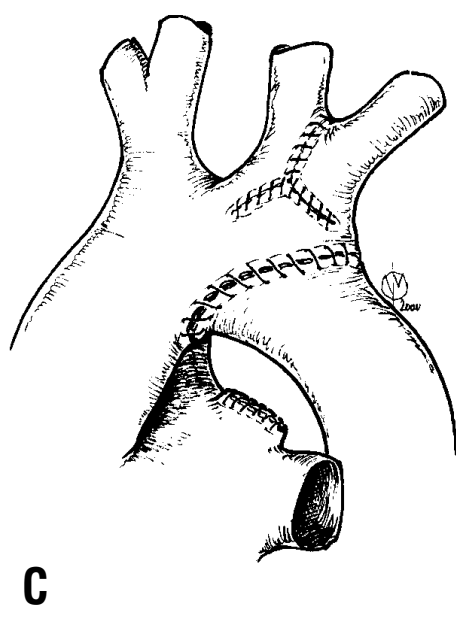

Fig 2. A, B, and C, Technical sequence of the procedure.

analysis of these patients revealed the crestlike protrusion of the superior arch wall into the lumen between the origins of the left common carotid and left subclavian arteries (Fig 1, A and $B$ ). The mean age at operation was $14.8 \pm 5$ days, ranging from 1 to 74 days. Twenty-two patients underwent concomitant pulmonary banding because of associated large ventricular septal defects. In all cases, the mean clamp time was less than 17 minutes. All patients have made an excellent recovery without any postoperative neurologic deficit. Recurrent aortic coarctation distal to the origin of the left subclavian artery developed during the follow-up period (mean $15 \pm 6$ months) in 6 patients. The recurrence was successfully corrected by balloon dilatation. No residual gradients across the reconstructed aortic arch segment were detected at periodic postoperative echocardiographic examinations with this technique.

Surgical technique. The surgical approach was through a left posterolateral thoracotomy in the fourth intercostal space. The aortic arch, proximal portions of the brachiocephalic vessels, patent ductus arteriosus, and upper half of the descending aorta were completely mobilized. The site of isthmic coarctation was first ligated, and the suture was pulled out through the thoracotomy and fixed to operating cloths to allow the arch and proximal portions of arch vessels to come down into the operative field to facilitate the procedure. A proximal curved clamp occluded the left carotid artery, the left subclavian artery, and the transverse arch between the innominate and the left common carotid arteries. The patent ductus arteriosus continued to perfuse the descending aorta during the time needed to enlarge the distal arch. An incision was made on the superior aspect of the distal aortic arch between the origins of the left common carotid and left subclavian arteries and then extended to the lateral aspects of both vessels (Fig 2, A). The length of these arteriotomies depended on the degree of distal arch stenosis and therefore on the expected aortic arch diameter after reconstruction. An
8-0 monofilament suture (Premium; Péters Lab, Bobigny, France) was then placed separately at the midpoint of each of the posterior lips of both arteriotomies to approximate and tie them to the midpoint of the posterior lip of the aortotomy performed between both vessels. Apposition surfaces between the left common carotid artery and distal arch on one side and between the left subclavian artery and the distal arch on the other side were sewn together starting from the midpoint of the posterior lip of the aortotomy and finishing at both extremities with a running 8-0 monofilament suture. Both remaining halves of the posterior lips of the left common carotid and left subclavian arteriotomies were sewn together with an 8-0 running monofilament suture starting from the midpoint of the aortotomy and finishing upward at the top of both arteriotomies (Fig 2, B).

The same procedure was repeated between the anterior lips of both arteriotomies and the midpoint of the anterior lip of the aortotomy and then between the remaining halves of the anterior lips of both arteriotomies. Another curved clamp was then placed on the descending aorta to apply traction, pulling the descending aorta upward to the site of anastomosis. The ductus arteriosus was ligated, the coarctation excised, and the undersurface of the aortic arch incised up to the left carotid artery with two appropriate counterincisions on the anterior and posterior aspects of the descending aorta. End-to-end anastomosis was performed beginning posteriorly with a running 7-0 polydioxanone suture (Fig 2, C) (PDS; Ethicon, Inc, Somerville, NJ).

Comment. Several kinds of aortic arch reconstruction techniques for isthmic coarctation associated with aortic arch hypoplasia involving one or all arch segments have been the subject of numerous publications. However, observations are usually focused on the location, extension, and diameter of the hypoplastic segment, and not on its morphologic characteristics. ${ }^{2,3}$ A segment of the aortic arch is hypoplastic if its length exceeds $5 \mathrm{~mm}$ and its diameter is less than $50 \%$ of the 
diameter of the ascending aorta or, more conveniently, of the descending aorta. ${ }^{4}$ However, for many years, we have observed that various anatomic patterns of the aortic arch can coexist with isthmic coarctation in an impressive number of patients. The most striking of these anatomic patterns that could have hemodynamic impact is the crestlike protrusion of the superior arch wall into the aortic lumen between the origins of the left common carotid and left subclavian arteries. This condition was first described and surgically treated in 2 patients by Amato, Rheinlander, and Cleveland. ${ }^{1}$ They used a similar technique consisting of longitudinally incising the left common carotid and the left subclavian arteries in the form of a $\mathrm{V}$ and then sewing them together by starting at the midpoint of the $\mathrm{V}$ incision to approximate the anterior and posterior walls of both arteries. Concomitant surgical repair of coarctation and distal arch stenosis lowers the arm-leg blood pressure gradients that can sometimes persist after conventional resection, subclavian flap repair, or even after extended endto-end coarctation repair, due to the above-described undiagnosed anatomic variant of distal arch hypoplasia. ${ }^{5}$

Midterm follow-up echocardiograms of our patients benefiting from this technique revealed harmonious remodeling and preserved growth characteristics of all involved structures. Compared with reversed subclavian flap angioplasty described for surgical correction of arch coarctation, this technique avoids sacrificing the left subclavian artery and the subsequent impaired blood supply to the left arm. Moreover, the diameter of the distal arch can be accordingly enlarged by placing the first stitches on the appropriate points selected on the posterior and anterior lips of both arteriotomies, bringing them to the midpoint of the aortotomy. This advantage is lost with reversed subclavian flap angioplasty, in which the diameter of the left subclavian artery determines the diameter of the reconstructed distal arch.
In conclusion, we believe that the management of a substantial number of patients in whom this anatomic arch pattern coexists with isthmic coarctation will depend on the awareness of the examiner. That treatment must provide appropriate surgical correction for definitive anatomic arch repair to reduce the incidence of failures resulting in unacceptable arm-leg pressure gradients and the prevalence of chronic arterial hypertension.

Received for publication July 12, 2000; accepted for publication Nov 6, 2000.

Address for reprints: A. Kalangos, $\mathrm{MD}, \mathrm{PhD}$, Clinic for Cardiovascular Surgery, University Hospital of Geneva, 24, rue Micheli-du-Crest, 1211 Geneva 14 Switzerland (E-mail: Afksendyios.Kalangos@hcuge.ch).

\section{REFERENCES}

1. Amato JJ, Rheinlander HF, Cleveland RJ. A method of enlarging the distal transverse arch in infants with hypoplasia and coarctation of the aorta. Ann Thorac Surg 1977;23:261-3.

2. Elliott MJ. Coarctation of the aorta with arch hypoplasia: improvements on a new technique. Ann Thorac Surg 1987;44: 321-4.

3. Hart JC, Waldhausen JA. Reversed subclavian flap angioplasty for arch coarctation of the aorta. Ann Thorac Surg 1983;36:715-7.

4. Zannini L, Gargiulo G, Albanese SB, Santorelli MC, Frascaroli G, Picchio FM, et al. Aortic coarctation with hypoplastic arch in neonates: a spectrum of anatomic lesions requiring different surgical options. Ann Thorac Surg 1993;56:288-94.

5. Vouhé P, Trinquet F, Lecompte Y, Vernant F, Roux PM, Touati G, et al. Aortic coarctation with hypoplastic aortic arch: results of extended end-to-end aortic arch anastomosis. J Thorac Cardiovasc Surg 1988;96:557-63. 